INVITED REVIEW

\title{
Immunosuppressive therapy in patients with thyroid eye disease: an overview of current concepts
}

\author{
G E Krassas and A E Heufelder ${ }^{1}$ \\ Department of Endocrinology and Metabolism, Panagia Hospital, Thessaloniki, Greece and ${ }^{1}$ Department of Gastroenterology, Endocrinology \\ and Metabolism, Philipps University, Marburg, Germany \\ (Correspondence should be addressed to G E Krassas, Department of Endocrinology and Metabolism, 'Panagia' General Hospital, \\ Tsimiski 92 - Thessaloniki 546 22, Greece; Email: Krassas@the.forthnet.gr)
}

\begin{abstract}
Thyroid eye disease (TED) is a debilitating disease impairing the quality of life of affected patients. Treatment is often not satisfactory. This review summarizes the existing literature and discusses the most widely used forms of treatment for TED such as glucocorticoids (GCs), and other immunosuppressive agents. GCs are the most commonly used treatment in patients with TED. Other immunosuppressive agents such as cyclosporin A, azathioprin, cyclophosphamide and ciamexone have been used, but the results are modest at best and indicate an unfavorable benefit-risk relationship. Limited experience indicates that methotrexate may be effective even in patients with refractory TED. Somatostatin analogs, octreotide and lanreotide, may provide a valuable, although costly, therapeutic alternative to GCs. Orbital radiotherapy has been used in the management of TED for almost 60 years. However, its beneficial effects have been questioned recently by several studies, the details of which have not yet been published. Other studies have argued in favor of orbital radiotherapy; however, the benefits appear to be limited to improvement of extraocular muscle dysfunction.
\end{abstract}

European Journal of Endocrinology 144 311-318

\section{Introduction}

Thyroid eye disease (TED) is an inflammatory condition of the orbits and the most frequent extrathyroidal complication of Graves' disease $(1,2)$. It is an organspecific autoimmune disease that is characterized by enlargement of the extraocular muscles and increased retrobulbar fatty/connective tissue, which cause exophthalmos, periorbital swelling, and venous congestion, the main clinical manifestations of the disease.

Involvement of orbital soft tissues, cornea, and optic nerve may occur during the natural history of the disease (3). Identification of patients with TED is based primarily on clinical signs and symptoms affecting one or both eyes (4). Additional information can be obtained from newer imaging methods such as magnetic resonance imaging (MRI) or computed tomography $(\mathrm{CT})$, which identify gross changes in the retrobulbar space such as muscle swelling, edema, and sometimes fibrosis $(2,5,6)$.

The pathogenesis of TED is almost certainly multifactorial. During the early stages of the disease, macrophages, highly specialized $\mathrm{T}$ cells, mast cells, and occasional plasma cells infiltrate the orbital connective, adipose, and muscle tissues $(7,8)$. Activation of $\mathrm{T}$ cells directed against a thyroid follicular cell antigen(s) that then recognizes and binds to a similar antigen(s) in orbital tissue is a probable but yet unproven theory (9). Alternatively, macrophages and dendritic cells may nonspecifically initiate the orbital immune response, which is then propagated by recruitment of sensitized $\mathrm{T}$ cells.

Several cytokines have been associated with the evolution of the orbital tissue changes in TED $(10,11)$. These include interferon $\gamma$ (IFN $\gamma$ ) (12), tumor necrosis factor- $\alpha$ (TNF- $\alpha)$, interleukin-1 (IL-1), and transforming growth factor- $\beta$ (TGF- $\beta$ ) (13) as well as other growth factors such as insulin-like growth factor-I (IGF-I) $(14,15)$ and platelet-derived growth factor $(16$, 17). These compounds are now known to be produced both by infiltrating immunocompetent cells and by residential fibroblasts, adipocytes, myocytes, and microvascular endothelial cells. These cytokines and growth factors stimulate cell proliferation, glycosaminoglycan (GAG) synthesis, and expression of immunomodulatory molecules in orbital fibroblasts and microvascular endothelial cells $(2,17-19)$. An increase in connective tissue and extraocular muscle volume within the bony orbits caused by accumulating hydrophilic compounds (predominantly GAG, the hydrophilic nature of which can attract water by osmosis) leads to the clinical 
manifestations of TED and causes proptosis, extraocular muscle dysfunction, and periorbital edema $(2,3)$.

It is frequently impossible to define the activity of the pathologic process and particularly the presence or absence of fibrosis upon clinical ocular examination $(1,2)$. Thus, it is sometimes difficult to distinguish the clinically active inflammatory stages of the disease from immunologically inactive, often fibrotic stages of the disease. Accurate assessment of the immunological activity of ophthalmopathy at the time of initial presentation is essential in order to chose the optimal therapeutic approach, as immunosuppressive therapy is beneficial mainly during the earlier active stages, whereas surgery represents the treatment of choice for advanced, inactive stages of the disease (9).

\section{Glucocorticosteroids: dinosaurs are still the prime choice}

In the absence of better alternatives of proven efficacy, immunosuppressive treatment of TED still largely rests with the use of glucocorticosteroids (GCs). Glucocorticosteroids exert a plethora of rather nonselective immunoregulatory and immunosuppressive actions; for example, they interfere with T- and B-lymphocyte functions, inhibit mononuclear cell recruitment to areas of inflammation, downregulate the expression of endothelial and extravascular adhesion molecules, and inhibit or modulate the production and release of various chemokines, cytokines, and arachidonic acid metabolites. Many of their effects are mediated through an inhibition of NFкB (20). In the context of TED, GCs have been shown to inhibit expression of human leukocyte antigen-DR (HLA-DR) and adhesion molecules by orbital fibroblasts, to decrease orbital fibroblast-associated metabolic activities such as cell proliferation as well as synthesis and release of GAG, and to suppress the production and release of various cytokines (21-23). Oral GCs have been employed for the management of active TED for more than four decades, and their efficacy is well documented in the literature. Since 1958, at least 14 published studies have reported an average response rate of $63 \%$ (range: $40-100 \%$ ) in 212 patients treated with oral glucocorticosteroids (24). Unfortunately, oral GCs therapy is frequently accompanied by unpleasant and potentially dangerous side effects, resulting in significant treatment-induced morbidity and poor patient compliance. Moreover, suppression of the inflammatory activity of TED by GCs is rarely long lasting once treatment doses have been tapered off. Finally, GCs have been shown to promote adipogenesis both in vitro and in vivo, which may be detrimental if it occurs within the orbits in patients with TED.

\section{Are intravenous glucocorticosteroids the way to go?}

Intravenous administration of GCs was initiated as a treatment for TED in the late 1980s. This was prompted by preliminary reports suggesting improved efficacy and reduced side effects of the intravenous as compared with the oral route. Since then, at least 11 studies have assessed the role of intravenous pulse therapy in TED (24). The average response rate was 77\% (range: $33-100 \%$ ) in 157 patients treated with intravenous glucocorticosteroids (24). From these studies, it appears that intravenous glucocorticoid pulse therapy exerts fewer side effects and is indeed better tolerated by patients than oral glucocorticoid therapy. Hyperglycemia, hypertension, infections, gastrointestinal problems, electrolyte disturbances, and psychiatric complications are the main side effects reported, but these appear to be rare events. Although significantly more laborious and costly to administer, the efficacy of intravenous bolus therapy with GCs may be superior to conventional oral treatment. This may be particularly relevant in patients with optic neuropathy, when a rapid treatment effect is urgently needed. However, the results of a randomized clinical trial directly comparing the two routes of GCs administration are still awaited. Because different regimes of drug administration, different dosages, and different associated treatments were used in the various studies, it is still difficult to conclude what intravenous GCs pulse doses and treatment intervals should be chosen and how treatment should be continued orally after the intravenous treatment phase. Furthermore, the potential advantages of the intravenous route of administration have to be weighed against a small but potentially serious risk of hepatotoxicity, as suggested by a recent report of a patient with TED who died from hepatic failure following intravenous glucocorticoid pulse therapy (25). Although underlying viral or autoimmune hepatitis or concomitant treatment with antithyroid agents may have increased the risk of hepatotoxicity, the etiology of this rare but serious side effect remains uncertain and worrisome.

\section{Other immunosuppressive agents: a wide choice of unproven options}

Other immunosuppressive agents, such as cyclosporin A, azathioprin, chlorambucil, cyclophosphamide, and ciamexone, are either ineffective in TED or carry an unfavorable benefit-risk relationship (24). Low-dose treatment with methotrexate is being used with apparent success even in patients with refractory TED (A E Heufelder, H D Schworm \& G Heufelder, unpublished observations); however, data from prospective clinical studies to conclusively prove its efficacy are still lacking. Given the striking similarities of the pathogenesis of rheumatoid arthritis (RA) and TED (26) and the 
fact that methotrexate has become a standard form of treatment for RA, this interesting drug deserves to be examined in patients with TED in a randomized, controlled study.

\section{Orbital radiotherapy: newer studies cast doubts on standard procedure}

Orbital radiotherapy (OR) has been used in the management of TED for 60 years. The rationale for its use resides with its nonspecific anti-inflammatory effects, which may include an inhibition of survival and metabolic activities of both activated mononuclear cells and orbital fibroblasts (24). In addition, low doses of ionizing irradiation have recently been shown to stimulate, in orbital fibroblasts, the production and release of IL-1 receptor antagonist, a potent local inhibitor of the pluripotent pro-inflammatory IL-1 in TED (27). In a survey of 11 studies involving 351 patients with TED, beneficial effects of OR were reported in $65 \%$ of cases, a percentage similar to that observed with oral prednisone (24). Orbital radiotherapy has little if any effect on proptosis, but tends to improve soft tissue inflammatory changes and optic neuropathy, although this was not supported by a recent prospective controlled clinical trial (28). In this well-conducted study, a significant but modest beneficial effect was only observed with respect to extraocular muscle dysfunction. However, despite this small benefit concerning diplopia, the percentage of patients spared from corrective strabism surgery was rather modest, raising the question of whether the time and expense associated with this procedure justify the routine use of OR in patients with TED (28).

The efficacy of standard OR has recently been challenged by a randomized prospective clinical study conducted at Mayo Clinic, Rochester, USA by C A Gorman, the details of which have not yet been published. Therefore, the role of OR in TED will have to be reassessed once these data have become available. Moreover, several recent studies have suggested that low-dose OR may be at least as effective as conventional high-dose OR in patients with active TED. In a retrospective evaluation of high $(20 \mathrm{~Gy})$ and low (10 Gy) doses of OR in more than 100 patients, no significant differences in outcome were observed (29). Furthermore, a randomized trial by the same authors compared three different radiotherapy protocols in 62 euthyroid patients with moderately severe, clinically active TED and no optic nerve involvement (30). Orbital radiotherapy (telecobalt 60) was administered in 20 fractions of 1 Gy per week over 20 weeks (group A), in 10 fractions of 1 Gy per day (group B), or in 10 fractions of $2 \mathrm{~Gy}$ per day over two weeks (group C). A successful outcome was observed in 67\% (group A), $59 \%$ (group B), and 59\% (group C) of patients. In patients with moderately severe, active TED, the prolonged $1 \mathrm{~Gy} /$ week protocol appeared to be more effective and better tolerated than the shorter radiotherapy regimens, and both subjective and objective ophthalmic signs decreased most in groups A (1 Gy/ week) and B (1 Gy/day). These data are in agreement with those of a study by Ravin et al. (31), who treated 37 patients with 10 fractionated doses of 1 Gy only. Muscle function in subjects with eye muscle abnormalities improved but did not return to normal. Visual function improved in all nine patients with optic neuropathy, although one patient still required orbital decompression.

The benefits of conventional OR are further challenged by the results of a prospective, randomized study conducted at 8 centers in Germany (J Gerling, unpublished observations). However, as the results of this study have been reported in abstract format only, detailed analysis of the data has yet to be achieved.

Orbital radiotherapy has been used in association with systemic high-dose GCs to combine the more rapid action of GCs and the more persistent effect of irradiation (32). In two randomized, prospective studies, the Pisa group showed that combined therapy was more effective than either GCs (33) or orbital radiotherapy alone (34), suggesting a possible synergistic effect of these two forms of treatment. Moreover, Prummel et al. (35) reported that orbital radiotherapy and high-dose oral prednisone were equally effective in patients with moderately severe ophthalmopathy, although prednisone was slightly more effective in reversing soft tissue changes, and orbital radiotherapy was somewhat superior in improving eye muscle motility.

In general, orbital radiotherapy is usually well tolerated and safe (36), although transient exacerbation of orbital edema, conjuctival hyperemia, and chemosis may occur during the first week of treatment. Also, cataract formation is a well-known complication of lens irradiation (37), and its severity and progression are dose related (38). Radiation-induced retinopathy has been noted in patients treated with cumulative doses much higher than those commonly used for TED (39). Kriss et al. (40) reported no significant retinal damage in patients treated with $30 \mathrm{~Gy}$ or less. However, pre-existing diabetic retinopathy may increase the likelihood of further damage to the retina by radiation.

\section{Somatostatin and somatostatin receptors}

Somatostatin (SM) was originally detected as an inhibitor of the release of growth hormone $(\mathrm{GH})$ by the pituitary gland. It is produced in two biologically active forms: a 14 amino acid form (SM-14) and an amino-terminally extended 28 amino acid form (SM-28). Somatostatin is found throughout the human body, but mainly within the endocrine glands and nervous system. In the CNS, it can act as a neurohormone and neurotransmitter, whereas in peripheral tissues it regulates endocrine and exocrine secretions and acts as 
a modulator of motility in the gastrointestinal tract (41). In 1978, Schönbrunn and Tashjian (42) first described SM receptors (SM-Rs) in the rat pituitary tumor cell line $\mathrm{GH} 4 \mathrm{C} 1$. The genes for the family of SM-Rs have been cloned in recent years (43). These genes are located on different chromosomes, but have a high sequence homology (44). Until now, five subtypes of human SMRs have been identified, all with a high affinity for SM-14 and SM-28 (45). Different SM-Rs can be expressed in the same tissues in overlapping patterns. All SM-Rs are coupled to G-proteins in the cell membrane and generate a transmembrane signal after binding of SM or SM analogs (SM-a) (46).

\section{Clinical use of SM analogs in the treatment of TED}

Various SM-a have been developed and used in clinical practice because the short half-life of SM-14 makes it unsuitable for routine treatment. The most frequently used SM-a are octreotide and lanreotide. The half-life of octreotide is 90-120 min when administered subcutaneously, and the pharmacodynamic effect lasts for 8$12 \mathrm{~h}$ (47). Lanreotide, a new long-acting analog of SM provided in a slow-release formulation, is more active than natural SM and shows a much longer duration of action. It has been proved to be effective in the treatment of acromegaly and is well tolerated when administered twice or three times per month $(48,49)$. Moreover, the long-acting release formulation of octreotide (Sandostatin-LAR), which has recently become commercially available and can be administered once every 4 weeks intramuscularly, has also proved to be very effective in suppressing GH and IGF-I in acromegaly $(50,51)$.

The effects of octreotide and lanreotide were found to be mediated mainly through SM-Rs subtypes 2 and 5 (45). New subtype-specific SM-a and SM-a antagonists, as well as nonpeptide subtype-specific SM-a are currently being developed and are of particular interest for clinical application (52).

Recent studies have shown successful therapy with octreotide in patients with active TED. Chang et al. (53), in an uncontrolled study, reported that octreotide had a beneficial effect in 6 patients with TED. In one controlled study (54), we found that octreotide treatment had a beneficial effect in 12 patients with moderately severe TED. Moreover, we showed that the response to low-dose octreotide treatment $(300 \mu \mathrm{g}$ daily) in these patients was correctly predicted by octreoscan-111. We proposed that octreoscan may predict the effectiveness of treatment with nonradioactive octreotide. However, we have encountered a small number of patients with a negative octreoscan who still responded to octreotide therapy.

In an uncontrolled study (55), 10 patients were treated with octreotide $(0.3 \mathrm{mg} /$ day $)$ for 3 months; although the authors claimed that 8 patients responded to this treatment, a critical reappraisal of their data seems to suggest that no more than 5 patients experienced a real improvement in ocular conditions, proptosis being only minimally affected. This treatment was particularly successful, however, in patients with soft tissue involvement (class II or III) (55). In another uncontrolled study, Kung et al. (56) evaluated the usefulness of octreotide compared with GCs. These authors noted that GCs and octreotide treatment were able to decrease, to a similar extent, the palpebral aperture and activity score after 3 months, but overall activity scores were lower after GCs compared with octreotide treatment. In addition, only GCs treatment was able to reduce intraocular pressure and muscle size as documented by MRI (56). By contrast, neither octreotide nor GCs significantly improved proptosis, whereas glycosaminoglycan excretion was reduced after both treatments (56). Finally, an absence of a beneficial effect of octreotide treatment was reported by Durak et al. (57) in three patients with active Graves' ophthalmopathy despite using high doses of the drug ( $1 \mathrm{mg} /$ day $)$.

Recently, we administered lanreotide at a dose of $40 \mathrm{mg}$ every 2 weeks over a period of 3 months to five patients with moderately severe Graves' ophthalmopathy and a positive octreoscan (58). Four of the five patients showed significant improvement of clinical activity scores (CAS) in both eyes, and the remaining patient showed improvement in one eye. These data were confirmed in a very recent study in which octreoscans were repeated at the end of the third month of treatment and were found to be negative in all patients (59).

Despite these promising results, it must be stressed that most of the studies conducted to date were uncontrolled and have included only small numbers of patients. Thus, a randomized, placebo-controlled prospective clinical study is needed, and such a study is currently under way.

The exact mechanism of action of SM-a has not yet been fully clarified. Three main explanations can be offered. First, SM suppresses IGF-I activity, and inhibition of IGF-I-mediated effects may be a promising strategy for controlling the orbital inflammatory process and its deleterious consequences (60). A second possible mechanism of action is direct inhibition of the release of lymphokines from T-lymphocytes (61). Cytokines, such as IL- 1 , IFN- $\gamma$, and TNF- $\alpha$, are known to be produced by orbital macrophages, dendritic cells, and infiltrating activated lymphocytes. These pro-inflammatory mediators are thought to play an important role in triggering and perpetuating the cascade of reactions that occur in the retro-orbital space of TED and eventually lead to clinical disease through stimulation of GAG synthesis in orbital preadipocytes and fibroblasts $(2,3)$. In addition, several of these cytokines stimulate the expression of immunomodulatory proteins (HLA-DR, heat shock protein-72, 
and intercellular adhesion molecules) by orbital fibroblasts, thus aiding in the perpetuation of the autoimmune response in the orbital connective tissue (3, 17).

Finally, SM-a may act directly on target cells through specific cell surface receptors. Gene expression of SM-R $1-5$ has been analyzed in various orbital cell types. In these studies, Graves' orbital lymphocytes were found to express SM-R 1-5, whereas in TED, orbital adipose tissue RNA encoding SM-R $1-3$ and 5 was detected $(62,63)$. In TED, extraocular muscle revealed expression of SM-R 1 and SM-R 2 genes, whereas orbital fibroblasts appear to express SM-R 1-3, but not SM-R 4 or 5 (62). Taken together, these data suggest that SM-a may bind to certain SM-R on the surface of various orbital cell types, such as lymphocytes, fibroblasts, and muscle cells, thereby altering their immunologic and metabolic activities (63). However, it is likely that SM-a act through a combination of these effects.

\section{Cytokine antagonists: hope for the future}

As outlined above, cytokines are critically involved in the pathogenesis of TED. They act both by triggering and perpetuating the cascades of reactions occurring in the retro-orbital space and eventually leading to the clinical manifestations of the disease (17).

Currently, the use of soluble cytokine receptors, either natural or genetically engineered cytokine antagonists, and anti-inflammatory cytokines in the management of severe inflammatory disorders is being investigated. Modulators of cytokine activity can exert their effects through multiple different mechanisms (64). Until now, little information has been available concerning the efficacy of IL-1 antagonists in human disorders (65), although IL-1 receptor antagonist (IL1RA) has been reported to have favorable effects in glucocorticoid-resistant graft versus host disease (66) and in septic shock syndrome (67). Ongoing studies are focused on the use of these biological agents in the management of refractory rheumatoid arthritis and lupus nephritis (68). However, the question of whether and to what extent IL-1 antagonists can be considered an effective treatment for these diseases has not been addressed.

Tan et al. (69) demonstrated in vitro that soluble IL$1 R$ and IL-1RA can inhibit IL-1-stimulated GAG synthesis and secretion by retro-orbital fibroblasts derived from patients with TED and highlighted the possibility that IL-1 antagonists might be useful for the treatment and/or prevention of TED (69). Nevertheless, it remains to be proved whether this also occurs in vivo and, more importantly, whether this results in an amelioration of the clinical symptoms of the disease. At present, the only trial of presumed cytokine modulation in TED was a small pilot study using pentoxifylline. This agent has significant anti-cytokine activity and was shown to inhibit IL-1-, TNF- $\alpha-$, and IFN- $\gamma$-induced
HLA-DR expression and GAG synthesis in cultural orbital fibroblasts. Ten patients with moderately severe TED were given intravenous pentoxifylline for 10 days, followed by oral therapy for an additional 10 weeks (70). Eight of the ten patients treated showed some improvement of soft tissue swelling, but not of proptosis or extra-ocular muscle involvement. Serum GAG and TNF- $\alpha$ levels declined only in the responders. It has to be mentioned that the lack of a control group in this study makes the findings very difficult to interpret.

However, before we consider anticytokine treatment effective and valuable for TED, there are several reasons why some caution should be observed. First, it is important to realize that in some instances soluble receptors (and cytokine autoantibodies) may function as specific carriers of cytokines, thereby increasing their biological half-life and facilitating rather than neutralizing their activities in the body (64). Secondly, information concerning the long-term safety of such agents is still lacking, especially when they are administered parenterally for a prolonged period of time. In addition, the doses of these agents required to reach a therapeutically effective concentration in the retro-orbital space remains to be defined. Thirdly, the cost-benefit ratio of a therapeutic course with these drugs has not yet been evaluated. This is particularly important because these drugs are very costly, they need to be used for an extended period of time, and their main benefit may rest with prevention rather than treatment of the disease (69). Finally, these compounds must be compared with conventional therapies to determine whether they do, indeed, provide superior efficacy along with better tolerability.

Despite these reservations, cytokine antagonists urgently deserve evaluation as a novel and promising approach to the treatment of TED. Clearly, further studies are needed to assess their safety, to determine their effective doses, and to evaluate the cost-benefit ratio of these new agents. Still, it has to be remembered that this therapeutic approach does not address the underlying basic mechanism of the disease. Unless an etiological treatment can be designed, we cannot claim that a fully satisfactory therapeutic approach to the disease has been achieved.

\section{Conclusions}

TED is an organ-specific autoimmune disease that is characterized by an enlargement of extraocular muscles and by an increase of retrobulbar connective tissue and fat. The pathogenesis of the disease is multifactorial. Several cytokines and growth factors, as well as various types of immunocompetent cells, have been detected within the orbital tissues in TED. These cytokines and growth factors stimulate cell proliferation, GAG synthesis, and expression of immunomodulatory molecules in orbital fibroblasts and microvascular endothelial cells. The increased 
accumulation of hydrophilic compounds, predominantly hydrophilic GAG, within the body orbit eventually leads to the clinical manifestations of TED. It has been suggested that immunosuppressive therapy is beneficial only during the earlier active stages of the disease, whereas surgery represents the treatment of choice for the inactive stages of the disease.

Glucocorticosteroids are still the most widely used treatment in patients with thyroid ophthalmopathy, and they exert a plethora of nonspecific immunoregulatory and immunosuppressive actions. Other immunosuppressive agents such as cyclosporin A, azathioprin, cyclophosphamide, and ciamexone have been used in the treatment of patients with TED, but the results are, at best, modest and carry an unfavorable benefit-risk relationship. In a preliminary study, methotrexate, the treatment standard for RA, appears to be effective even in patients with refractory TED; however, data from prospective, controlled clinical trials are needed to prove its efficacy. Orbital radiotherapy alone or in combination with systemic high dose GCs has been used in the management of TED for almost 60 years. Recent studies and data have suggested that lowdose OR may be at least as effective as conventional high-dose OR in such patients. Moreover, its beneficial effects have been questioned recently by several studies, the details of which have not yet been published and, therefore, cannot be evaluated.

In recent years, several studies have shown promise with the long-acting SM-a octreotide and lanreotide in patients with active TED. SM-a may provide a welltolerated, although costly, therapeutic alternative to GCs, especially in patients who experience significant side effects on GCs. However, data from prospective placebo-controlled studies with larger numbers of patients are needed before their role in the treatment of TED can be defined.

\section{References}

1 Jacobson DH \& Gorman CA. Endocrine ophthalmopathy: current ideas concerning etiology, pathogenesis and treatment. Endocrine Reviews 19845 200-220.

2 Burch HB \& Wartofsky L. Graves' ophthalmopathy: current concepts regarding pathogenesis and management. Endocrine Reviews 199314 747-793.

3 Bahn RS \& Heufelder AE. Pathogenesis of Graves' ophthalmopathy. New England Journal of Medicine 1993329 1468-1475.

4 Moncayo R, Baldisserra I, Decristoforo C, Kendler D \& Donnemiller E. Evaluation of immunological mechanisms mediating thyroid-associated ophthalmopathy by radionuclide imaging using the somatostatin analog ${ }^{111}$ In-octreotide. Thyroid $1997721-29$

5 Trokel SL \& Jacobiec FA. Correlation of CT scanning and pathologic features of ophthalmic Graves' disease. Ophthalmology $1981 \mathbf{8 8} 553-564$.

6 Kahaly G, Diaz M, Just M, Beyer J \& Lieb W. Role of octreoscan and correlation with MR imaging in Graves' ophthalmopathy. Thyroid 19955 107-111.

7 Tallstedt L \& Norberg R. Immunohistochemical staining of normal and Graves' extraocular muscle. Investigative Ophthalmology and Visual Science 198829 175-184.
8 Perros P \& Kendall-Taylor P. Pathogenesis of thyroid-associated ophthalmopathy. Trends in Endocrinology and Metabolism 19934 270-275.

9 Burch HG, Gorman CA, Bahn RS \& Garrity JA. Ophthalmology. In Werner and Ingbar's The Thyroid. A Fundamental and Clinical Text, 8th edition, pp 531-548. Eds L Braverman \& RD Utiger. Philadelphia: Lippincott-Williams \& Wilkins, 2000.

10 Natt N \& Bahn RS. Cytokines in the evolution of Graves' ophthalmopathy. Autoimmunity 199726 129-136.

11 Bahn RS. Cytokines in thyroid eye disease: potential for anticytokine therapy. Thyroid $1998 \mathbf{8} 415-418$.

12 Smith TJ, Bahn RS, Gorman CA \& Cheavens M. Stimulation of glycosaminoglycan accumulation by interferon gamma in cultured retro-ocular fibroblasts. Journal of Clinical Endocrinology and Metabolism 199172 1169-1171.

13 Korducki J, Loftus S \& Bahn R. Stimulation of glycosoaminoglycan production in cultured human retro-orbital fibroblasts. Investigative Ophthalmology and Visual Science 199233 209-214.

14 Hansson HA, Petruson B \& Skottner A. Somatomedin C in the pathogenesis of malignant exophthalmos of endocrine origin. Lancet $19861218-219$.

15 Weightman DR, Perros P, Sherif IH \& Kendall-Taylor P. Autoantibodies to IGF-I binding sites in thyroid-associated ophthalmopathy. Autoimmunity 199316 251-257.

16 Heufelder AE \& Bahn RS. Modulation of Graves' orbital fibroblast proliferation by cytokines and glucocorticoid receptor antagonists. Investigative Ophthalmology and Visual Science 199435 $120-127$.

17 Heufelder AE. Pathogenesis of Graves' ophthalmopathy. Recent controversies and progress. European Journal of Endocrinology 1995132 532-541.

18 Heufelder AE \& Scriba P. Characterization of adhesion receptors on cultured microvascular endothelial cells derived from the retro-orbital connective tissue of patients with Graves' ophthalmopathy. European Journal of Endocrinology 1996134 51-60.

19 Metcalfe RA \& Weetman AP. Stimulation of extraocular muscle fibroblasts by cytokines and hypoxia: possible role in thyroidassociated ophthalmopathy. Clinical Endocrinology 199440 6772.

20 Liden J, Rafter I, Truss M, Gustafsson JA \& Okret S. Glucocorticoid effects on NF-kappaB binding in the transcription of the ICAM-1 gene. Biochemical and Biophysical Research Communications 2000 273 1008-1014.

21 Heufelder AE, Bahn RS \& Smith TJ. Regulation of glucocorticoids of interferon gamma-induced HLA-DR antigen expression in cultured human orbital fibroblasts. Clinical Endocrinology 1992 37 59-63.

22 Heufelder AE, Wenzel BE \& Bahn RS. Glucocorticoids modulate the synthesis and expression of a $72 \mathrm{kDa}$ heat shock protein in cultured Graves' retro-ocular fibroblasts. Acta Endocrinologica 1993128 41-50.

23 Heufelder AE \& Bahn RS. Modulation of Graves' orbital fibroblast proliferation by cytokines and glucocorticoid receptor agonists. Investigative Ophthalmology and Visual Science 199435 120-127.

24 Bartalena L, Pinchera A \& Marcocci C. Management of Graves' ophthalmopathy: reality and perspectives. Endocrine Reviews 200021 168-199.

25 Weissel M \& Hauff W. Fatal liver failure after high-dose glucocorticoid pulse therapy in a patient with severe thyroid eye disease. Thyroid 200010521.

26 Bahn RS. Understanding the immunology of Graves' opththalmopathy. Is it an autoimmune disease? Endocrinology and Metabolism Clinics of North America 200029 287-296.

27 Muhlberg T, Joba W, Spitzweg C, Schworm HD, Heberling HJ \& Heufelder AE. Interleukin-1 receptor antagonist ribonucleic acid and protein expression by cultured Graves' and normal orbital fibroblasts is differentially modulated by dexamethasone and irradiation. Journal of Clinical Endocrinology and Metabolism 2000 $85734-742$.

28 Mourits MP, van Kempen-Harteveld ML, Garcia MB, 
Koppeschaar HP, Tick L \& Terwee CB. Radiotherapy for Graves' orbitopathy: randomized placebo-controlled study. Lancet 2000 355 1505-1509.

29 Kahaly GJ, Roesler HP, Kutzner J, Pitz S, Mueller-Forell W, Beyer J et al. Radiotherapy for thyroid-associated orbitopathy. Experimental and Clinical Endocrinology and Diabetes 107 Suppl 51999 S201-S207.

30 Kahaly GJ, Rosler HP, Pitz S \& Hommel G. Low versus high dose radiotherapy for Graves' ophthalmopathy: a randomized, single blind trial. Journal of Clinical Endocrinology and Metabolism 2000 85 102-108.

31 Ravin JC, Sisson JC \& Knapp WT. Orbital radiation for the ocular changes of Graves' disease. American Journal of Ophthalmology 197579 285-288.

32 Bartalena L, Marcocci C \& Pinchera A. Treating severe Graves ophthalmopathy. Bailliere's Clinical Endocrinology and Metabolism $199711521-530$.

33 Bartalena L, Marcocci C, Chiovato L, Laddaga M, Lepri G, Andreani D et al. Orbital cobalt irradiation combined with systemic corticosteroids for Graves' ophthalmopathy: comparison with systemic corticosteroids alone. Journal of Clinical Endocrinology and Metabolism 198356 1139-1144.

34 Marcocci C, Bartalena L, Bogazzi F, Bruno-Bossio G, Lepri A \& Pinchera A. Orbital radiotherapy combined with high-dose systemic glucocorticoids for Graves' ophthalmopathy is more effective than radiotherapy alone: results of a prospective study. Journal of Endocrinological Investigation 1991 14 853-860.

35 Prummel MF, Mourits MP, Blank L, Berghout A, Koornneef L \& Wiersinga WM. Randomized double-blind trial of prednisone versus radiotherapy in Graves' ophthalmopathy. Lancet 1993 342 949-954.

36 Wiersinga W \& Prummel MF. Retrobulbar radiation in Graves' ophthalmopathy. Journal of Clinical Endocrinology and Metabolism $199580345-347$.

37 Pinchera A, Bartalena L, Chiovato L \& Marcocci C. Radiotherapy of Graves' ophthalmopathy. In The Eye and Orbit in Thyroid Disease, pp 301-316. Eds CA Gorman, RR Waller \& JA Dyer. New York: Raven Press, 1984.

38 Merriam GR \& Focht EF. A clinical study of radiation cataracts and the relationship to dose. American Journal of Röentgenology 195777 759-785

39 Kinyoun JL, Kalina RE, Brower SA, Mills RP \& Johnson RH. Radiation retinopathy after orbital irradiation for Graves' ophthalmopathy. Archives of Ophthalmology $19841021473-$ 1476

40 Kriss JP, McDougall IR \& Donaldson SS. Graves' opthalmopathy. In Current Therapy in Endocrinology, pp 104-109. Eds DT Krieger \& W Bardin. New York: Decker, 1983-1984.

41 Van Hagen PM, Kuijpers RWAM, Mooy CM, Froger CL, Kwekkeboom DJ \& Baarsma GS. Perspectives on somatostatin and somatostatin receptor expression in autoimmune disease of the eye. In Octreotide: The Next Decade, pp 291-306. Ed SWJ Lamberts. Bristol, UK: BioScientifica, 1999.

42 Schönbrunn A \& Tashjian H Jr. Characterization of functional receptors for somatostatin in rat pituitary cells in culture. Journal of Biological Chemistry 1978213 6473-6483.

43 Humphrey P \& Reisine T. Somatostatin receptor nomenclature consensus. Ciba Foundation Symposium 1995190 261-262.

44 Patel YC, Greenwood MT, Panetta R, Hukovic N, Grigorakis S, Robertson L et al. Molecular biology of somatostatin receptor subtypes [Review]. Metabolism 199645 31-38.

45 Patel YC \& Srikant CB. Subtype selectivity of peptide analogs for all five cloned human somatostatin receptors (SSTr 1-5). Endocrinology $19941352814-2817$

46 Florio T \& Schettini G. Multiple intracellular effectors modulate physiological functions of the cloned somatostatin receptors. Journal of Molecular Endocrinology 199617 89-100.

47 Pless J. Chemistry and pharmacology of sandostatin. Zeitschrift für Gastroenterologie 28 Suppl 21990 4-7.

48 Caron P, Morange-Ramos I, Cogne M \& Jaquet P. Three years follow-up of acromegalic patients treated with intramuscular slow-release lanreotide. Journal of Clinical Endocrinology and Metabolism 199782 18-22.

49 Colao A, Marzullo P, Ferone D, Marin V, Pivonello R, Di Somma C et al. Effectiveness and tolerability of slow release lanreotide treatment in active acromegaly. Journal of Endocrinological Investigation 199922 40-47.

50 Flogstad AK, Halse J, Bakke S, Lancranjan I, Marbach P, Brüns Ch et al. Sandostatin LAR in acromegalic patients: long term treatment. Journal of Clinical Endocrinology and Metabolism 1997 82 23-28.

51 Newman CB, Melmed S, George A, Torigian D, Duhaney M, Snyder $\mathrm{P}$ et al. Octreotide as primary therapy for acromegaly. Journal of Clinical Endocrinology and Metabolism $1998 \mathbf{8 3} 3034$ 3040.

52 Rohrer SP, Birzin ET, Mosley RT, Berk SC, Hutchins SM, Shen DM et al. Rapid identification of subtype-selective agonists of the somatostatin receptor through combinatorial chemistry. Science $1998282737-740$.

53 Chang TC, Kao SCS \& Huang KM. Octreotide and Graves' ophthalmopathy and pretibial myxoedema. British Medical Journal $1992 \mathbf{3 0 4} 158$.

54 Krassas GE, Dumas A, Pontikides N \& Kaltsas Th. Somatostatin receptor scintigraphy and octreotide treatment in patients with thyroid eye disease. Clinical Endocrinology 199542 571-580.

55 Ozata M, Bolu E, Sengul A, Tasar M, Beyhan Z, Corakci A et al. Effects of octreotide treatment on Graves' ophthalmopathy and circulating sICAM-1 levels. Thyroid 19966 283-288.

56 Kung AWC, Michon J, Tai KS \& Chan FL. The effect of somatostatin versus corticosteroid in the treatment of Graves ophthalmopathy. Thyroid $19966318-324$.

57 Durak I, Durak H, Ergin M, Yurekli Y \& Kaynak S. Somatostatin receptors in the orbits. Clinics in Nuclear Medicine 199520237 242.

58 Krassas GE, Kaltsas Th, Dumas A, Pontikides N \& Tolis G. Lanreotide in the treatment of patients with thyroid eye disease. European Journal of Endocrinology 1997136 416-422.

59 Krassas GE, Doumas A, Kaltsas Th, Halkias A \& Pontikides N. Somatostatin receptor scintigraphy before and after treatment with somatostatin analogues in patients with thyroid eye disease. Thyroid $1999947-52$.

60 Hansson HA, Petruson B \& Skottnerm A. Somatomedin C in pathogenesis of malignant exophthalmos of endocrine origin. Lancet $1218-219$.

61 Malec P, Zeman K, Markiewicz K, Tchorzewski H, Nowak Z \& Baj Z. Short term somatostatin infusion affects T-lymphocyte responsiveness in humans. Immunopharmacology 19891745 49.

62 Heufelder AE. Somatostatin analogues in Graves' ophthalmopathy. Journal of Endocrinological Investigation 199720 suppl to no 7 50-52.

63 Krassas GE. Somatostatin analogues in the treatment of thyroid eye disease. Thyroid $1998 \mathbf{8} 443-445$.

64 Bartalena L, Marcocci C \& Pinchera A. Editorial: Cytokine antagonists: new ideas for the management of Graves' ophthalmopathy. Journal of Clinical Endocrinology and Metabolism 1996 81 446-448.

65 Debets R \& Savelkoul HFJ. Cytokine antagonists and their potential therapeutic use. Immunology Today 199415 455458.

66 Antin JH, Wenstein HJ, Guinan EC, McCarthy P, Bierer BE, Gilliland DG et al. Recombinant human interleukin-1 receptor antagonist in the treatment of steroid-resistant graft versus host disease. Blood $1994841342-1348$.

67 Fisher CJ Jr, Dhainaut JF, Opal SM, Pribble JP, Balk RA, Slotman GJ et al. Recombinant human interleukin-1 receptor antagonist in the treatment of patients with sepsis syndrome. Journal of American Medical Association 1994271 1836-1843.

68 Kalder JR. Biologic agents in the therapy of inflammatory rheumatic diseases, including therapeutic antibodies, cytokine and cytokine antagonists. Current Opinion in Rheumatology 1994 $6281-286$. 
69 Tan GH, Dutton CM \& Bahn RS. Interleukin-1 (IL-1) receptor antagonist and soluble IL-1 receptor inhibit IL-1induced glycosaminoglycan production in cultured human orbital fibroblasts from patients with Graves' ophthalmopathy. Journal of Clinical Endocrinology and Metabolism $1996 \mathbf{8 1}$ 449-452.

70 Balazs C, Kiss E, Vamos A, Molnar I \& Farid NR. Beneficial effect of pentoxifylline on thyroid-associated ophthalmopathy. Journal of Clinical Endocrinology and Metabolism 199782 1999-2002.

Received 6 September 2000

Accepted 21 December 2000 